\title{
Design of a compact high-power neutron source-The EURISOL converter target
}

\author{
K. Samec*, R.Ž. Milenković, S. Dementjevs, M. Ashrafi-Nik, A. Kalt \\ Paul Scherrer Institut, Villigen, 5232 Villigen, Switzerland
}

\section{A R T I C L E I N F O}

Article history:

Received 27 February 2009

Received in revised form

9 April 2009

Accepted 28 April 2009

Available online 14 May 2009

Keywords:

Neutron spallation target

Liquid metal

Isotope production facility

\begin{abstract}
A B S T R A C T
The EURISOL project, a multi-lateral initiative supported by the EU, aims to develop a facility to achieve high yields of isotopes in radioactive beams and extend the variety of these isotopes towards more exotic types.

The neutron source at the heart of the projected facility is designed to generate isotopes by fissioning uranium carbide (UC) targets arranged around a $4 \mathrm{MW}$ neutron source. For reasons of efficiency, it is essential that the neutron source be as compact as possible, to avoid losing neutrons by absorption whilst maximising the escaping neutron flux, thus increasing the number of fissions in the UC targets. The resulting configuration presents a challenge in terms of absorbing heat deposition rates of up to $8 \mathrm{~kW} / \mathrm{cm}^{3}$ in the neutron source; it has led to the selection of liquid metal for the target material.

The current paper presents the design of a compact high-power liquid-metal neutron source comprising a specially optimised beam window concept. The design is based on two-dimensional (2D) and three-dimensional (3D) computational fluid dynamics (CFD) numerical simulations for thermal hydraulics and hydraulic aspects, as well as finite-element method (FEM) for assessing thermomechanical stability. The resulting optimised design was validated by a dedicated hydraulic test under realistic flow conditions. A full-scale mock-up was built at the Paul Scherrer Institute (PSI) and was tested at the Institute of Physics of the University of Latvia (IPUL).
\end{abstract}

(c) 2009 Elsevier B.V. All rights reserved.

\section{Introduction}

The development of neutron sources has progressed in recent years in response to the demand for ever-increasing levels of neutron flux, currently $10^{14} \mathrm{n} / \mathrm{cm}^{2} / \mathrm{s}$ at the surface of the source. Such sources rely on a physical phenomenon known as a spallation reaction, wherein a high-energy proton beam impacts a heavy target element such as lead or mercury, leading to the emission of a large number of high-energy neutrons. Typically 20-30 neutrons can be emitted per incoming proton, depending on the energy of the proton beam, the nature of the target material and the overall design of the source.

The Paul Scherrer Institute (PSI) operating the Swiss Spallation Neutron Source SINQ has extensive experience with acceleratordriven neutron sources and liquid-metal (LM) technology. The idea of developing a LM target was instigated in the late 1980s by PSI, where considerable experimental work was carried out [1]. The SINQ neutron source was originally intended as a LM target with a cusp design for the window and a naturally recirculating

\footnotetext{
* Corresponding author.

E-mail address: karel.samec@psi.ch (K. Samec).
}

LM flow entrained by placing the heat source, i.e. the beam deposition at the bottom of the LM target. However in the end, the relatively immature state of LM technology prevented such an advanced design from emerging and a solid target was preferred instead.

More recently, there has been a renewed interest in LM for the development of new types of nuclear reactors, such as the hybrid/ ADS type or the Gen IV reactor for which six different concepts, mostly with LM, are being considered.

The ADS concept for transmutation of long-lived radioactive waste was the driving force behind the MEGAPIE project, a joint initiative by six European research institutions, the EU, JAEA (Japan), KAERI (Korea) and the DOE (USA), which has recently tested the world's first high-power LM spallation target on the basis of lead-bismuth eutectic (LBE) [2].

The MEGAPIE target operated successfully in SINQ for 4 months at a power level of $0.8 \mathrm{MW}$. After this first success, the PSI-SINQ team proceeded with the development of a new liquid-metal neutron source operating at a far higher power density in the context of the EURISOL project. This initiative was proposed by an EU consortium of research establishments comprising notably the CERN [3].

The peak power density in the LM target material is an order of magnitude higher in EURISOL compared to MEGAPIE. The increase 
in power prompted a change in the design of the beam window, a thin solid wall through which the proton beam penetrates to impact the LM target material. This aspect of the program was the focus of much attention and critical to the validation of a safe window design.

Development of LM targets has in the past relied on prior water tests with transparent plexiglass models to visualise the complex fluid dynamic phenomena essential for evacuating large amounts of heat from a confined space. The MEGAPIE project and associated qualification experiments demonstrated that advances in computational fluid dynamics (CFD) allowed accurate predictions to be made of the flow pattern in LM; [4] the resulting thermal-hydraulic behaviour correlated well with experimental data, in particular those collected during the MEGAPIE irradiation period. Therefore, the EURISOL project decided to do without any water tests using plexiglass models and to rely purely on CFD for optimising the source. At the end of the design study, a fullscale model was built and tested with LM in order to validate the design directly. The benefit in speeding up development was considered to outweigh the potential risk of failing in the final test. Furthermore it was felt that a successful outcome would reinforce the case for using CFD computational tools for all manners of liquid-metal applications not limited to spallation sources.

\section{The EURISOL converter target design}

The analysis in the following sections demonstrates the proposed design's advantages in terms of cooling the point of entry of the beam and improving overall hydraulic performance. Obtaining experimental proof of these advantages was the goal of the hydraulic test of the full-scale mock-up.

Table 1

Baseline design parameters.

\begin{tabular}{ll}
\hline Parameter & Value \\
\hline Target material & Mercury \\
Target diameter $(\mathrm{cm})$ & $<15$ \\
Target length $(\mathrm{cm})$ & $>40$ \\
Beam particle & Proton \\
Beam energy $(\mathrm{Gev})$ & 1 \\
Beam current $(\mathrm{mA})$ & 4 \\
Beam power $(\mathrm{MW})$ & 4 \\
Gaussian width $(\mathrm{mm})$ & $15-25$ \\
\hline
\end{tabular}

\subsection{Design parameters}

The EURISOL project development team at CERN carried out initial concept-level studies of the entire facility to find the best possible configuration for the production of high yields of isotopes [3]. The resulting design parameters are contained in Table 1 . The most constraining parameter, the heat deposition from the proton beam, is shown in Fig. 1; axial and radial profiles are given for a beam width of $\sigma=15 \mathrm{~mm}$.

Early on in the development of the program, mercury was chosen as the target material in order to generate a harder neutron flux than with the LBE used in MEGAPIE. Mercury has the advantage of being liquid at room temperature unlike LBE, which is also hazardous from a radio protection point of view, as LBE produces polonium when impacted by protons. The main disadvantage of mercury is that, unlike LBE, it boils at low temperature, $350^{\circ} \mathrm{C}$ at atmospheric pressure, or $457^{\circ} \mathrm{C}$ at $5 \times 10^{5} \mathrm{~Pa}$ static pressure, the reference pressure chosen for the EURISOL loop. Calculations showed that in the event of the mercury flow being instantaneously stopped by a valve failure or debris, there would be only $0.7 \mathrm{~s}$ in which to shut down the beam to avoid pressure rupture of the target hull. A fast beam interrupt system of the type used for MEGAPIE in SINQ [5] would however eliminate this risk.

The material selected for the hull and overall structure of the target was T91 steel, since it was used with success in the lower portion of MEGAPIE, where it was highly irradiated. The mock-up tested in the Institute of Physics of the University of Latvia (IPUL) was made of stainless-steel 316 , which was more readily available, yet has similar thermo-mechanical properties outside irradiation.

\subsection{Target design}

Successive iterations, detailed in the following sections, resulted in the design of the EURISOL converter illustrated in the top of Fig. 2. The figure shows the internal structure of the target; the proton beam penetrates into the LM through the conical cusp hull window on the right; the LM enters the target through the inlet below left, reverses at the window and exits the target on the left, downstream of the main guide tube. The main features of the design are the window, which is shaped as an inverted conical cusp, the flow vanes at the front end of the target and the guide tube in the form of a venturi. The whole structure is about $1 \mathrm{~m}$ in length and $15 \mathrm{~cm}$ in diameter.

The assembly sequence is illustrated in Fig. 2. Despite its novel design, EURISOL was manufactured using established techniques, a challenge given its complexity. The sequence for assembling and manufacturing the target starts with the insertion of the guide
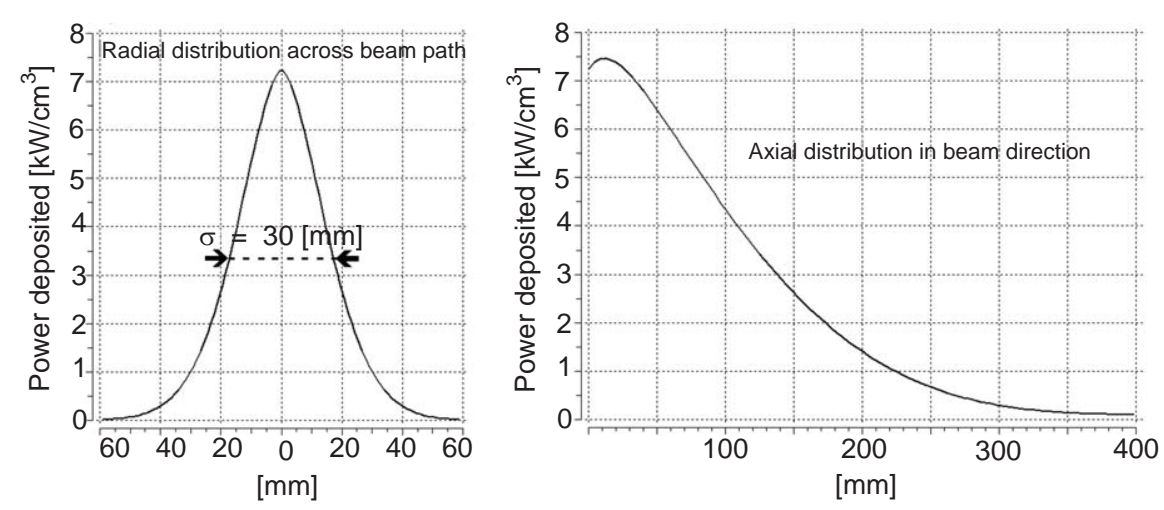

Fig. 1. Beam heat deposition $\left(\mathrm{kW} / \mathrm{cm}^{3}\right)$. 


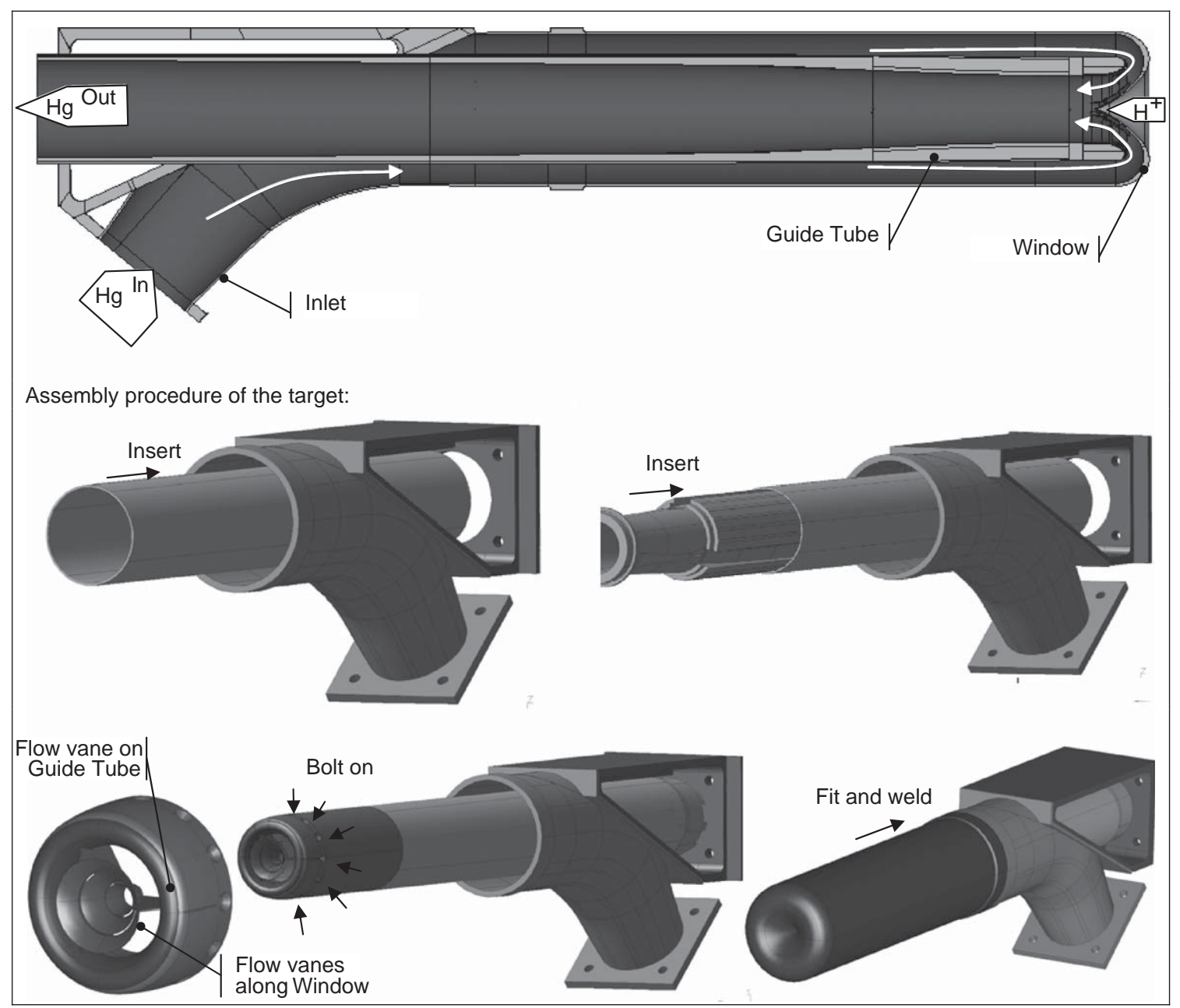

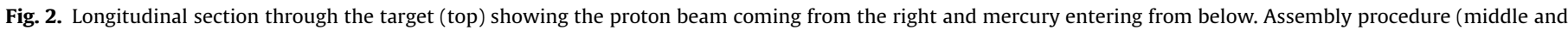
bottom) of the proposed design.

tube into the inlet section (Fig. 2 middle). The guide tube contains grooves suitable for routing measurement cables, thermocouple leads and pressure taps. In the next step, the guide tube nozzle comprising flow vanes is fixed to the guide tube. The nozzle can also be manufactured without flow vanes; an advantage for assessing their effect in the test. Finally the external hull comprising the cusp window is welded to the inlet using electronbeam welding. A bolted interface at the rear establishes all in- and out-flowing LM connections to the loop. The actual mockup tested at IPUL is shown in Fig. 3; it incorporated a few modifications to the proposed design shown in Fig. 2, which were necessary to facilitate the acquisition of data during the test.

\subsection{Initial design iterations: the apex of the cusp window}

The apex of the cusp window is a critical aspect of the design. At this point, the beam penetrates the target wall precisely where the flow reverses through $180^{\circ}$ (Fig. 2, top). The inverted conical shape of the cusp window serves to reverse the flow back from the point of entry of the beam, thus carrying away the high heat deposition from the beam whilst wetting the surface of the beam window with cool LM. The main parameter influencing the temperature distribution and stress in the window is its shape. Hence, in a first approach [6], stresses on the window apex were assessed with a simplified axis-symmetric section model of the apex of the cusp window in two dimensions using the commercial ANSYS software. Fig. 4a shows the cusp apex model [7], in which the beam heat deposition was programmed as a volumetric function and the LM cooling was represented as a variable convection boundary condition. Results from the model indicated that cooling of the window could be enhanced by increasing the fluid velocity, which raises the heat transfer coefficient (HTC). A larger HTC signifies there is less thermal resistance at the interface between the wall and the fluid. The model showed that increasing the HTC was effective in reducing the window temperature up to an HTC value of $15,000 \mathrm{~W} / \mathrm{m}^{2} \mathrm{~K}$, at which point further increases in HTC did not yield any improvement. This limit for the HTC is achievable in LM if the velocity is kept above $3 \mathrm{~m} / \mathrm{s}$; below that limit there is a risk of overheating the window.

Temperatures are therefore held in check, as long as a steady flow of LM cools the apex of the cusp. Any detachment of the flow from the window would immediately cause rapid temperature variations, which would lead to thermal stress cycling (Fig. 4a, right).

The different geometric parameters such as the wall thickness distribution, the opening angle and curvature of the cusp window were programmed in APDL, or ANSYS Parametric Design Language (parameters in Fig. 4a, left), to arrive at an optimal shape in terms of temperature and stress whilst remaining within technological limits, i.e. a paper-thin window would be structurally impossible 

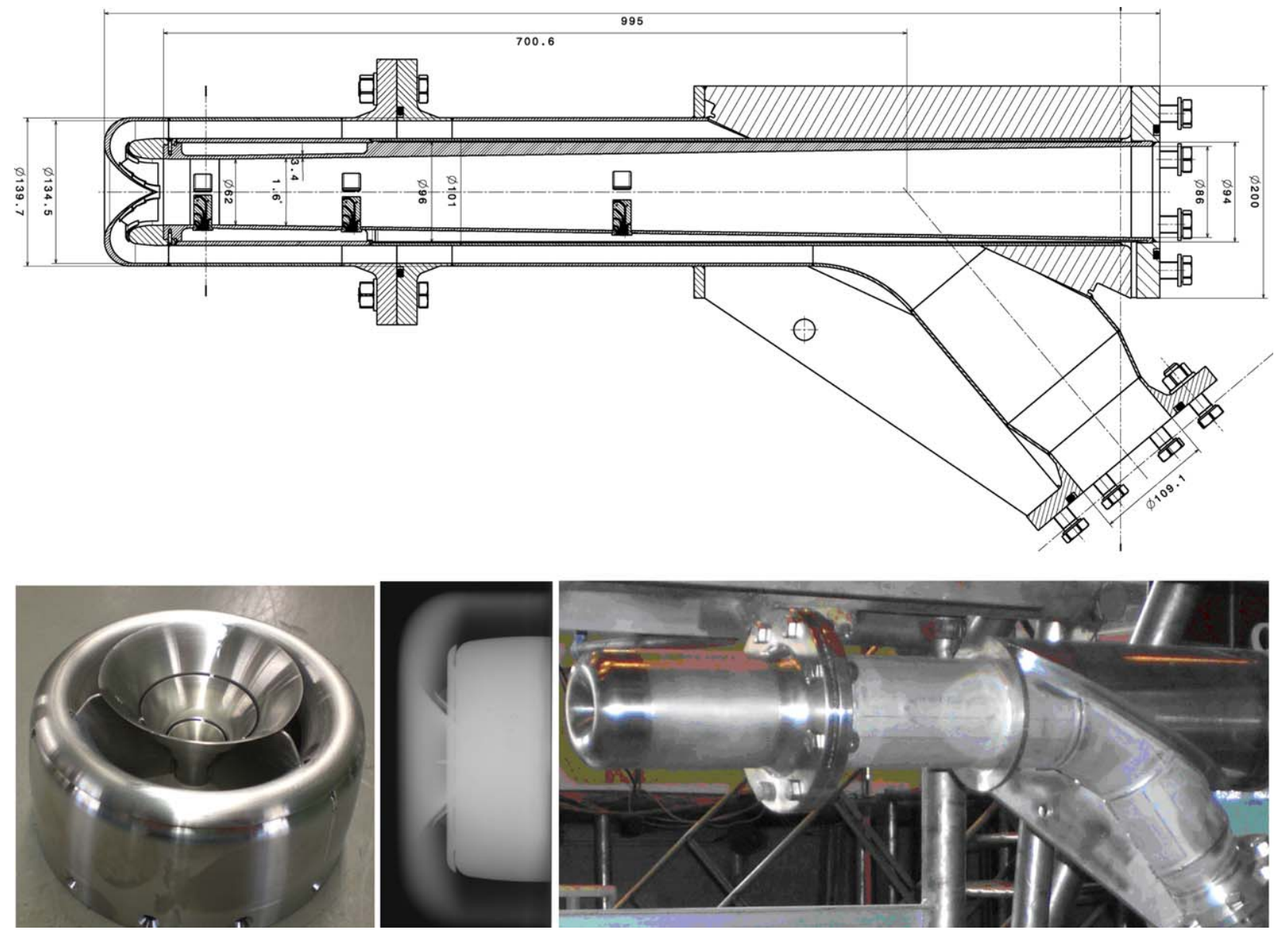

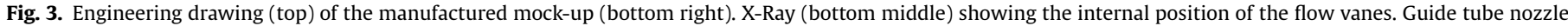
with flow vanes (bottom left).

to build and stabilise. The simplified cusp model showed that the thickness at the apex of the window had to be less than $0.8 \mathrm{~mm}$ for a beam width of $\sigma=15 \mathrm{~mm}$, a challenge in terms of manufacturing. However, if the beam width were to be relaxed from $\sigma=15$ to $25 \mathrm{~mm}$, the minimum thickness at the apex could be raised to $1.4 \mathrm{~mm}$.

\subsection{Subsequent design iteration step: window and guide tube}

The next step in the iteration consisted in integrating the thickness distribution of the cusp apex into a two-dimensional (2D) section CFD model of the full target as shown in Fig. 4b for the final optimised shape. Thus, the hydraulic design of the forward (beam) end of the target was optimised using an axissymmetric section model cut longitudinally through the target, the symmetry axis being the beam axis. This gradual approach allowed a large range of options to be studied in a reasonable time frame.

Although the MEGAPIE configuration was broadly retained in EURISOL, one of the main differences besides the window geometry was the change over to a horizontal configuration. Otherwise, as in MEGAPIE, the LM enters the target by an outer annulus, which sends the cool fluid towards a window. At this point it reverses at the window, which is also the point of entry of the beam and then flows back in the opposite direction down a guide tube located coaxially inside the outer annulus. A first calculation, featuring a simple straight cylinder for the guide tube, shown in Fig. 5 (left) revealed the presence of an extended recirculation pattern on the backswept portion of the internal guide tube. The recirculation is problematic as it would accumulate heat from the proton beam and lead to large eddies, which could provoke cyclical fatigue failure either directly through wall pressure variations or through thermal fatigue cycling. Furthermore, the energy lost to the vortex would result in a higher pressure loss, which would then need to be compensated for by the pump. In order to prevent this phenomenon, it seemed advisable to thicken the guide tube so as to give it a rounded leading edge, somewhat akin to the section of a wing. In the optimised shape, the vortex disappeared as shown by the streamline map in the right-hand side of Fig. 5 . An additional benefit was a greater acceleration of fluid over the window and a more homogeneous flow field downstream of the window, where most of the beam heat is deposited.

The initial CFD calculations also uncovered some instability of the flow at the tip of the window cusp. Detachment of the flow may occur at the critical point where the beam penetrates the target and heats up the wall. This weakness in the design was improved upon by including circular rings with profiles in the form of wings (Fig. 5 right), positioned close to the forward end of the guide tube, at the apex of the window in order to guide and stabilise the flow in these critical areas. The so-called flow vanes function in a manner similar to fowler flaps on aircraft wings, which help reattach the airstream in highly turbulent flight regimes occurring at high angles of attack. The characteristic target system temperatures and flow parameters resulting from the final design shown in Figs. 4b and 5 (right) are summed up in Table 2. 
a

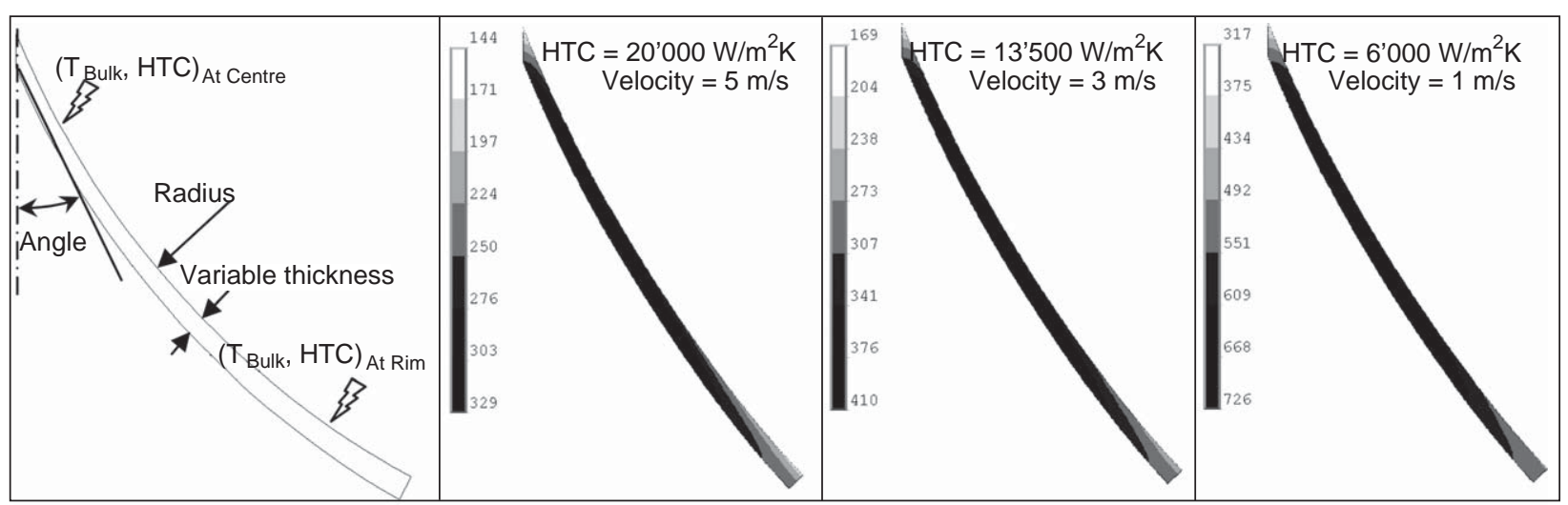

b

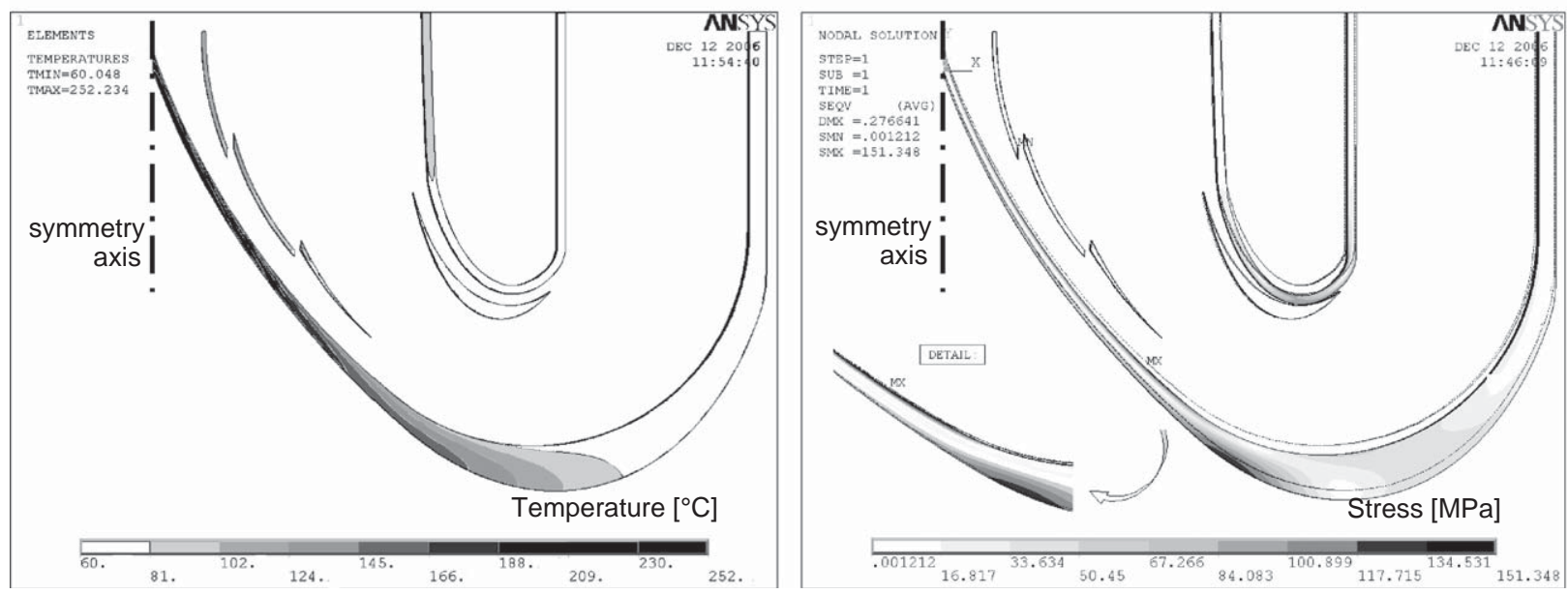

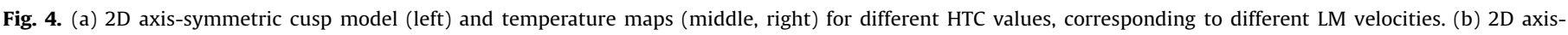
symmetric calculation of the temperature $\left({ }^{\circ} \mathrm{C}\right)$ (right) and stress (MPa) (left) in the forward end of the target for the optimised shape.

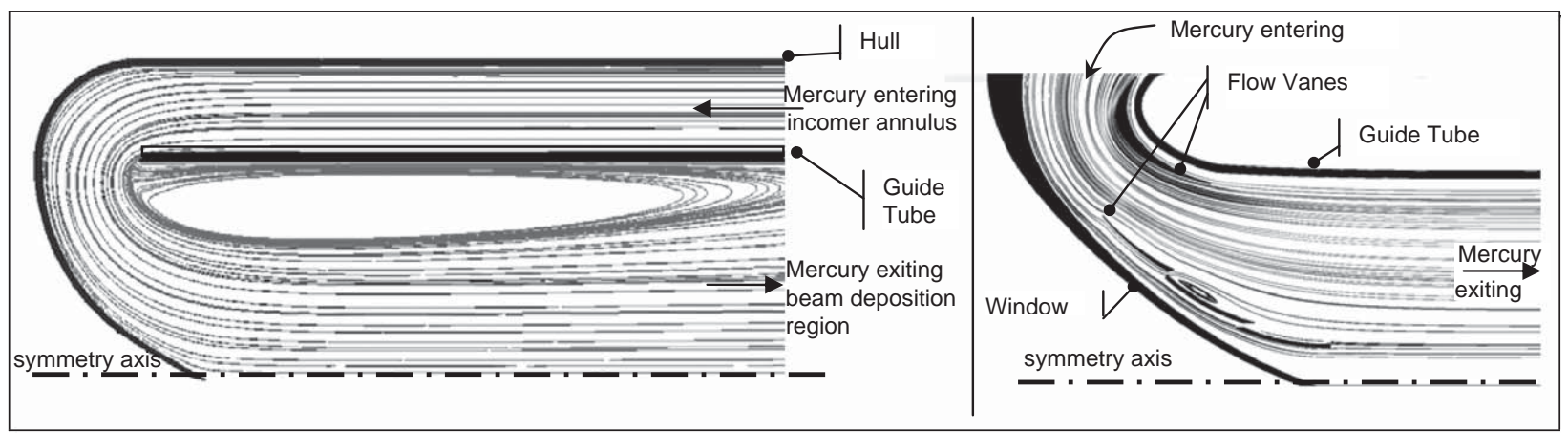

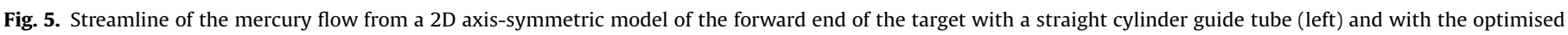
shape (right).

Stresses arising in the target are mostly the result of differential thermal expansion. This is particularly the case in the window, whose inner surface is wetted by cool incoming liquid mercury and whose outer surface facing the beam is exposed to the beam tube vacuum. Hence stresses are caused by the hotter outer surface expanding and thus being compressed and the cooler inner surface being stretched. The inner pressure residing in the target also adds stress, but is actually beneficial to the window as it compresses uniformly the apex, thus lowering the tensile stress on the inner surface, albeit at the cost of increasing the compressive stress on the outer surface. As compressive stresses do not lead to crack formation, the net overall effect of pressure is positive. A linear buckling analysis showed the window apex has a safety margin of 6 against buckling under a LM pressure of $10^{6} \mathrm{~Pa}$, which means the window apex could sustain $6 \times 10^{6} \mathrm{~Pa}$ internal pressure.

Further away from the central apex, the conical window becomes thicker and reaches a maximum thickness at the point where the concave cusp shape reverses and joins onto the outer annulus hull (Fig. 4b). This local thickening of the window adds 
Table 2

Target baseline characteristics.

\begin{tabular}{lc}
\hline Parameter & Value \\
\hline Temperature increase $\left({ }^{\circ} \mathrm{C}\right)$ & 120 \\
Mass flow rate $(\mathrm{kg} / \mathrm{s})$ & 171 \\
Reference static pressure (bar) & 5 \\
Pressure drop $($ bar) & 0.8 \\
Peak cavitation pressure (bar) & -2.6 \\
Peak temperature $\left({ }^{\circ} \mathrm{C}\right)$ & \\
Window & 252 \\
Guide tube & 120 \\
Flow vanes & 140 \\
Mercury & 261 \\
Peak velocity $(\mathrm{m} / \mathrm{s})$ & \\
Window & 3 \\
Incomer & 1.7 \\
Guide tube & 5 \\
\hline
\end{tabular}

stiffness, where it is needed to resist the internal pressure that bears down on the cusp and would otherwise tend to push it inside out. Only the section outside the main deposition area of the beam, a distance of more than $3 \sigma$ from the main axis, has been thickened; otherwise the thicker section would intersect the beam path and be subject to thermal stress.

\section{Results from 3D CFD modelling}

The previous iterations delivered an optimised shape, which was studied more closely in three dimensions in order to assess the likelihood of large turbulent structures forming inside the target. Particular emphasis was placed on the LM entering the target from below (Figs. 1 and 2). A succession of threedimensional (3D) CFD analyses followed a gradual approach aimed at limiting risk in the development [8]. First, the fore section of the target was modelled, including the flow vanes and the guide tube. Then, the inlet and incomer annulus were examined separately in three dimensions. Finally the entire model was set up from inlet to outlet (Fig. 7). All models comprised a $180^{\circ}$ segment, and thereby assumed half-symmetry about the vertical plane. This is a reasonable assumption, as the most likely cause for instability in the flow lies in the angled off-axis inlet pipe entering the target from below.

\subsection{Window model in $3 D$}

The 3D model of the window area of the target exceeds one million cells, and was implemented after the simpler 2D models had already delivered an optimal section shape. The 3D model of the window allowed non-axis-symmetric aspects such as the flow surrounding the supports of the flow vanes to be examined, which was not possible with the previous 2D models. It was also used to determine structural margins on the forward end of the target.

The turbulence model is a shear stress transfer (SST) function developed by Ansys-CFX, which has the advantage of promoting numerical stability, at the cost of smoothing out real instabilities in the flow. The stability of the SST turbulence model is due to automated wall functions, which account for the boundary layer. However the finer detail of a vortex street, such as may be shed by a vane support for instance, would not be resolved with this type of turbulence model. Further refinements are therefore needed, which are described in the following, in particular the LES turbulence model.
The impact of the beam on the window area was also examined in three dimensions with the 3D window model. The temperature distribution in the fluid and structure (Fig. 6) remained identical to that found in the 2D section model (Fig. 4), as the dissymmetry introduced by the three $120^{\circ}$ vane supports has little thermal inertia and no role in cooling.

The effect of the buoyancy force was studied, as the target is horizontal. There was no visible effect from integrating a buoyancy force into the model (Fig. 6); temperatures and flow speeds remained unchanged because of very high flow speeds reached in the target window.

The consequence of an off-axis proton beam was examined by shifting the beam axis in the formulation of the heat deposition by $5 \mathrm{~mm}$. In such a case, model results showed that the temperature gradient through the wall does not change compared with the symmetric case, leading to the same stress magnitude. The flow field was not affected by a shift in position of the beam, which confirms that buoyancy plays no role in the window area due to the high local fluid velocities.

\subsection{Inlet CFD model in $3 D$}

The entrance of fluid from a position below the target offset from the main axis of symmetry can potentially affect the balance of the flow further downstream in the target. In order to assess the likelihood of such imbalances, a dedicated model of the inlet section was set up that used both SST and $k-\varepsilon$ turbulence models. It served to investigate fully the development of the LM entering the target under a variety of conditions, representing various turbulence levels at the entrance and different hydraulic losses at the exit [9].

The study of the inlet focused on the development of the flow as it turns from the circular inlet and enters the incomer annulus. This was an important step to verify that the design could deliver stable flow conditions past the window and thereby ensure adequate cooling. The inlet study was able to show that an off-axis inlet does not disturb the balance between the LM flowing through the top and bottom part of the annulus just before the window. Indeed, there is no more than 5\% difference in flow distribution between the LM flowing at the top of the cusp entrance and that coming through the bottom part. Although a small and stable vortex is located at the junction of the inlet to the annulus it has no influence on the stability and symmetry of the flow further downstream. This is due to the fact that the incomer annulus has a length to hydraulic diameter ratio of over 30 sufficient for all imbalances in the distribution of LM between the top and bottom of the outer annular section to be equalised.

\subsection{Complete CFD model in three dimensions}

A complete CFD model of the target from inlet to outlet (Fig. 7) was assembled to gauge the likelihood of hydraulic instability propagating throughout the target. It assumed half-symmetry about the vertical plane. The symmetry plane was taken through the mid-plane of one of the three flow-vane supports spaced out at $120^{\circ}$ (insert in Fig. 8a). In doing so, the influence of the flow vanes on the fluid could be accounted for as well as any possible imbalance by the inlet offset. The model was run both with the flow vanes and without. The CFD model was restricted to the fluid domain. The structure was represented by infinitely stiff adiabatic walls. There was neither coupling between the fluid and the structure nor any heat transfer from the beam in order to sustain numerical stability and accelerate convergence. Hence this particular model did not look into the heating phenomena due to the beam, which had already been examined with the 3D window 


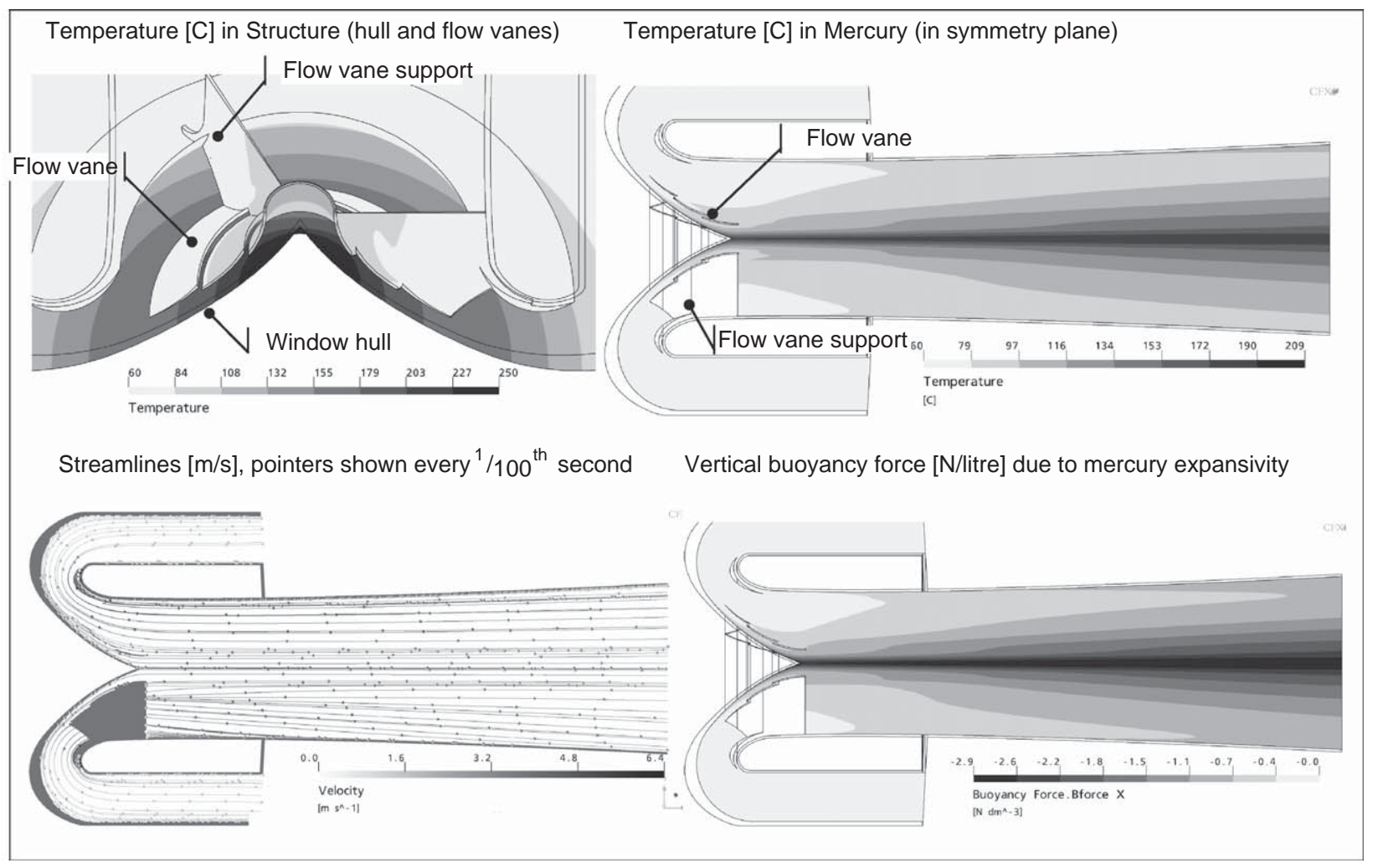

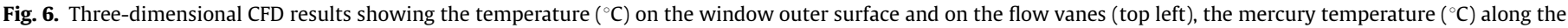
symmetry plane (top right), the streamlines along the symmetry plane (bottom left) and the buoyancy force ( $\mathrm{N} / \mathrm{litre}$ ) (bottom right).

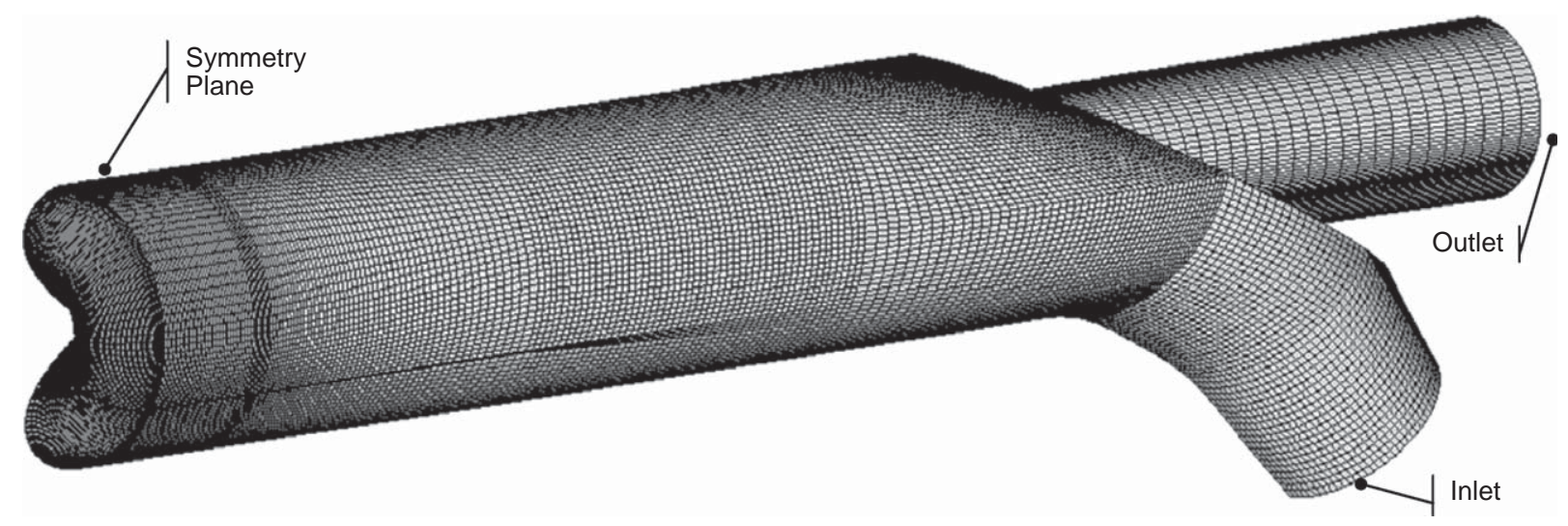

Fig. 7. Three-dimensional half-symmetry CFD model of the target.

model (Section 3.1 here above). Likewise, the issue of resonance was addressed separately with a dedicated dynamic structural model (Section 4 hereafter).

With three million cells defining a fully structured mesh, the global 3D CFD model was deemed sufficiently precise to assess large instabilities in the flow so as to prepare for the hydraulic test. The turbulence model used in the analysis was initially an SST function, which served to ramp up the flow from zero to full power $(12.6 \mathrm{l} / \mathrm{s})$ over a $10 \mathrm{~s}$ simulated time span. Once the full flow rate was reached, the simulation was restarted with a large eddy simulation (LES) turbulence model to investigate the stability of the flow and to ensure that no large periodically oscillating structure existed in the fluid. In spite of all the simplifications made to the model, the investigation with LES of such a complex flow demanded a time step of $10^{-4} \mathrm{~s}$ and indeed at times $10^{-5} \mathrm{~s}$; the computational effort was therefore considerable, requiring a 16-node cluster operating at $3 \mathrm{GHz}$.

The velocity profiles in Fig. 8a show a comparison of the version with and without flow vanes; in particular the velocity magnitude projected onto the vertical symmetry plane at a representative time when turbulence is fully developed. The lefthand figures illustrate the acceleration of fluid produced by the flow vanes at the forward end of the guide tube. The flow vanes close to the window appear able to attach the flow to the surface of the window. Indeed, unlike the model without vanes (righthand figure) there is a total absence of flow detachment on the window with the flow vanes. In addition, high-velocity zones in the guide tube towards the exit seem smaller with the vanes, thus decreasing the pressure variations along the inner surface of the target, which could lead to resonance. However the flow-vane 
a

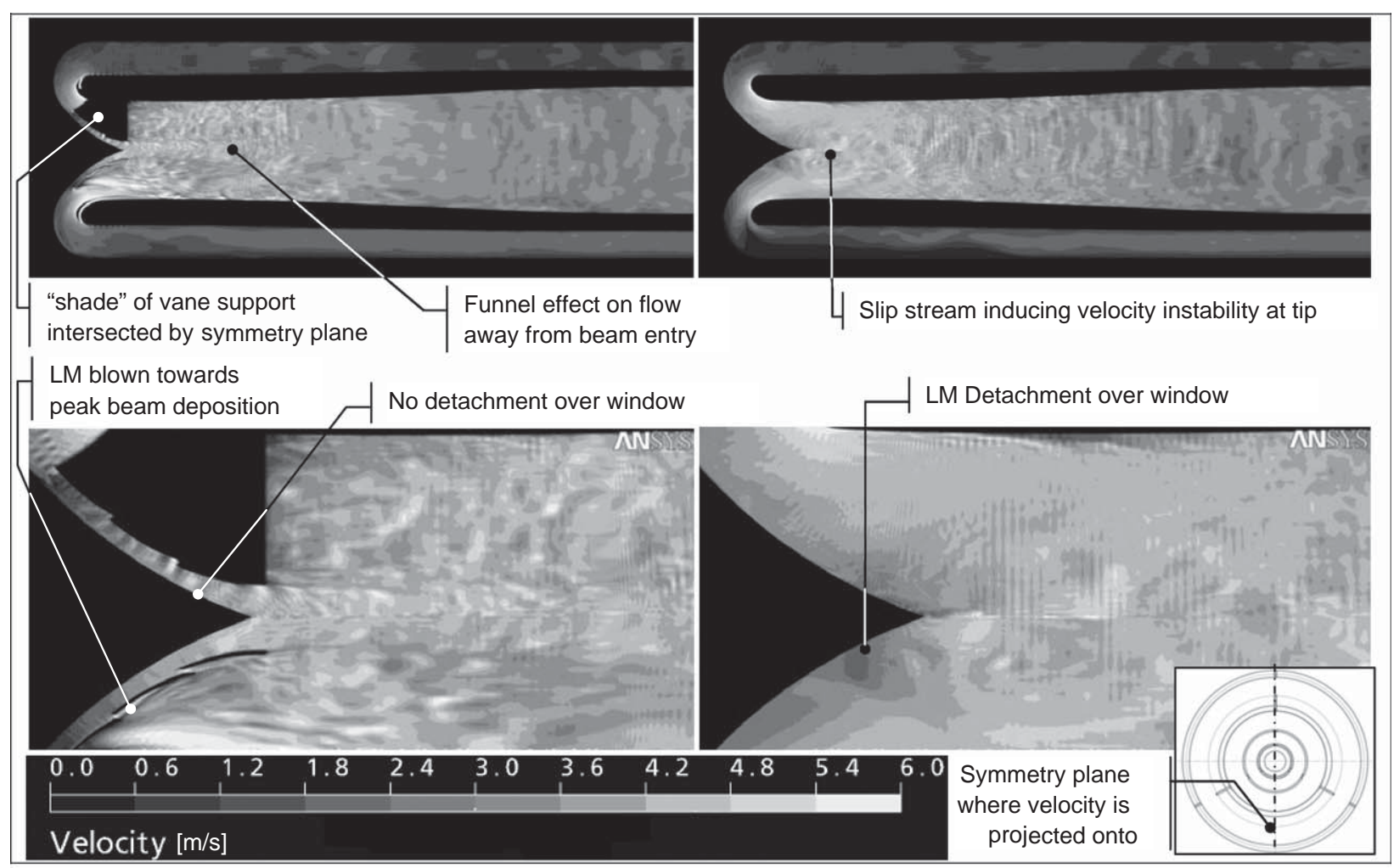

b

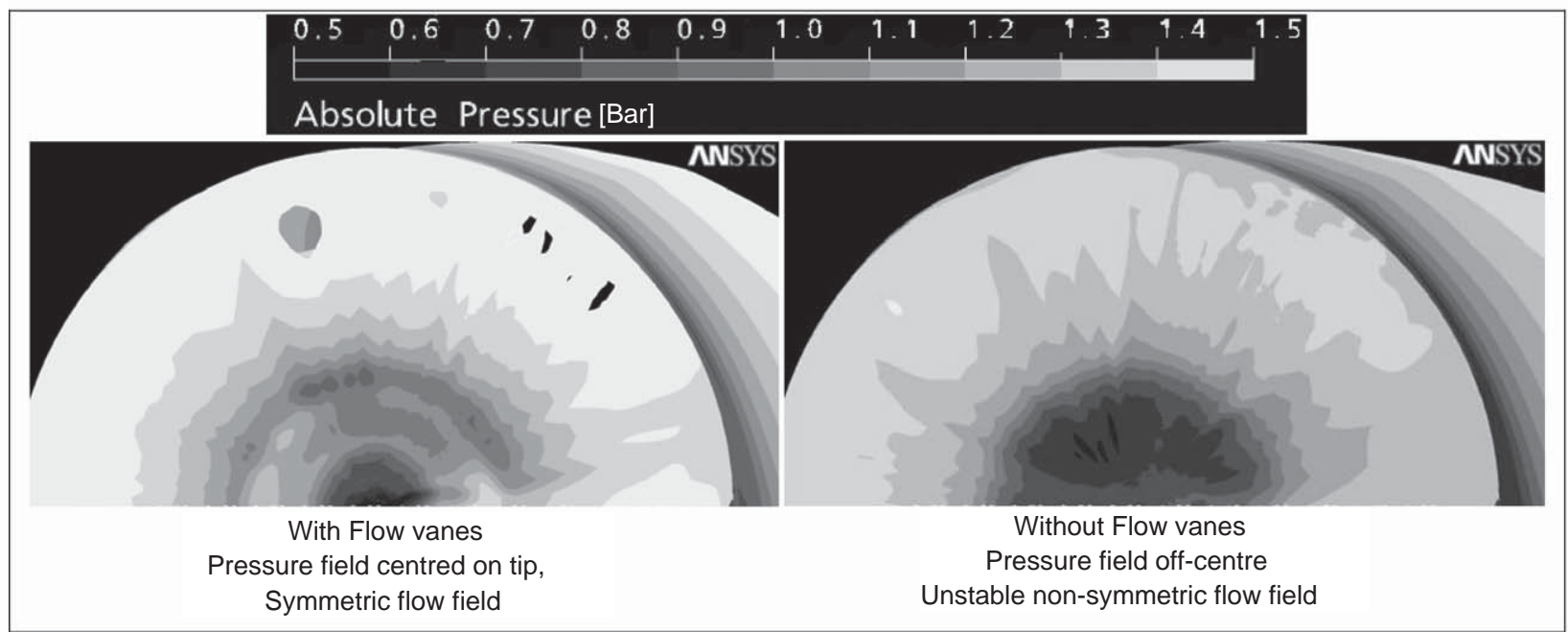

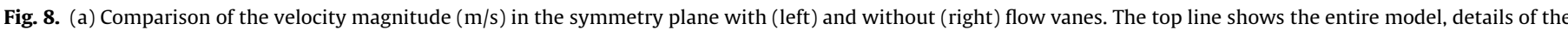
flow around cusp window are shown in the bottom. (b) Pressure on the window (bar) with (left) and without (right) flow vanes.

supports have some influence on the fluid distribution; a boundary layer develops off the leading edge of the supports; yet no Von Karman vortex street is shed. Drag is increased by the flow-vane supports, which add approximately $2 \times 10^{4} \mathrm{~Pa}$ of pressure drop. In a production model of the target, the vanes could be attached to the window rather than the guide tube thus lowering the section opposing the flow.

The pressure plots on the window in Fig. 8b also show an excerpt for a similar time. The left-hand plot for the version with the flow vanes has a symmetric pressure field located precisely over the apex of the cusp window at the beam entry point, whereas this area is less stable without the flow vanes as shown in the right-hand side of the figure. Pressure on the window is directly related to the local velocity on the wall and hence cooling ability. A stable symmetric pressure field is therefore a good indicator of the favourable flow conditions brought about by the flow vanes.

The likelihood of cavitation is increased by the flow vanes, which contain numerous sources for localised pressure drops. With flow vanes the over-pressure needed to contain cavitation was estimated to be $2.6 \times 10^{5}$ versus only $1 \times 10^{5} \mathrm{~Pa}$ without the flow vanes. An over-pressure in the target of 5 bar has been specified to increase the boiling temperature of the mercury, such that in both instances cavitation would be contained. 


\section{Structural calculations}

The major challenge in designing a compact target for $4 \mathrm{MW}$ of beam power lies in the very high velocities needed to evacuate large heat deposition densities. Mercury flowing at $6 \mathrm{~m} / \mathrm{s}$ poses a serious challenge in terms of structural stiffness as the target is essentially built in at one end, from which the mass is suspended.

Coupled resonance between the fluid turbulence inside the target and the natural resonance frequencies of the target must therefore be considered. In a simplified approach [10], the weight of the liquid mercury inside the target was added onto a structural finite-element method (FEM) model of the target as a surface mass, uniformly distributed on all wetted areas. The spatial pressure distribution on all surfaces wetted by LM was extracted from the complete 3D CFD calculation and projected onto the structural FEM model shown in Fig. 9, lower right. The pressure oscillations at representative points were extracted from the LES simulation and a discrete Fourier transform of the temporal variation of the pressure yielded the frequency-dependent amplitude of the pressure variation in a frequency range up to $200 \mathrm{~Hz}$. These dynamic loads were then entered into a sine-sweep harmonic analysis of the FEM structural model. The resulting stresses and displacements of the structure were calculated as shown in Fig. 9 for the flow vanes and the target hull.

The hull shows a characteristic frequency at only $40 \mathrm{~Hz}$, which is lower than the $60-70 \mathrm{~Hz}$ obtained from a purely structural eigen-value analysis. Coupling with the fluid is responsible for lowering the frequency; however the energy content is relatively low, as demonstrated by a peak displacement of only $0.05 \mathrm{~mm}$ at the tip of the hull (Fig. 9, upper right).

The window vanes on the other hand are subject to far higher dynamic loads, with a peak in the weld of the outermost flow vane reaching $125 \mathrm{MPa}$ at high frequency. Such amplitudes in a high- frequency domain are a concern as they may lead to high-cycle fatigue; the weld accumulates millions of cycles per hour of operation. Hence, although the existing flow vanes were judged adequate for a short hydraulic test, strengthening this part of the design is essential for a long-term operational target.

\section{Manufacturing of the prototype}

The prototype built for the full-scale test incorporated some modifications to the design originally proposed and calculated in the current paper. Such changes were necessary to accommodate manufacturing constraints, without jeopardizing the test and acquisition of data. The modifications are as follows:

- The inlet section was changed from an integral machined shape to a series of welded standard-size tubes, thus modifying the conduit shape and restricting the inlet and outer annulus section to $75 \%$ of the original one.

- The interchangeable guide tube nozzle was manufactured in two versions; with and without flow vanes to allow their effect to be studied.

- An additional support was inserted between the hull and the guide tube to stiffen the structure as a precaution against cavitation testing.

- The window cusp integrated to the fore hull was detachable thanks to a bolted flange interface to ease access to the internal organs of the target, in particular to allow pressure sensors to be installed and the guide tube nozzle to be exchanged. Obviously in a production neutron target neither a bolted flange interface nor the test instrumentation would be installed and the fore hull would be simply welded on.

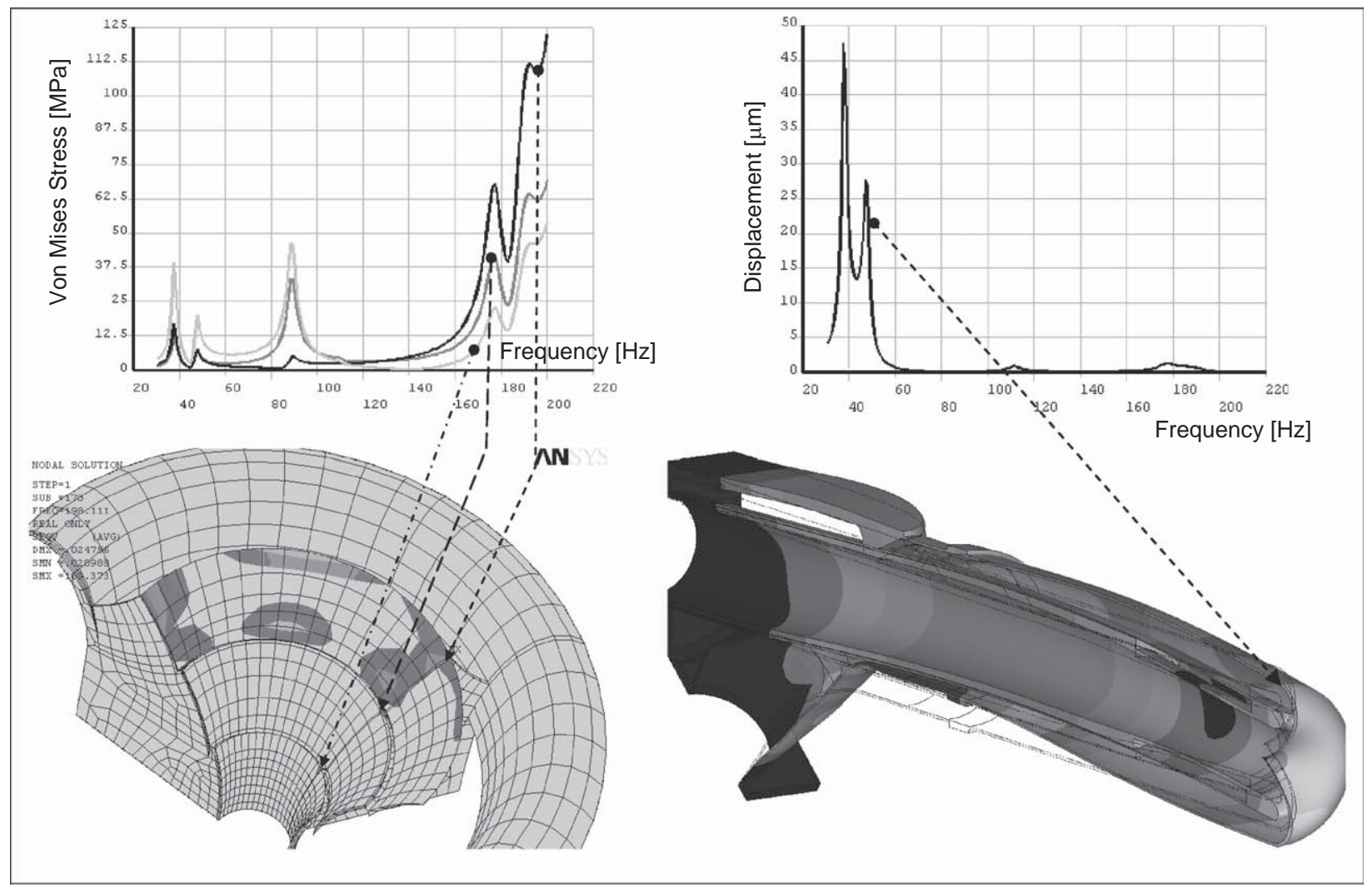

Fig. 9. Resonance modes of the flow vanes showing stresses in (MPa, left) and hull structure deformations in ( $\mu$ m, right). 
The prototype incorporating these modifications is shown in Fig. 3; it has successfully completed a series of hydraulic "cold" tests in the mercury loop at IPUL [11].

\section{Conclusion}

The development of a high-power neutron converter target in the context of the EURISOL initiative was a unique opportunity to push back the envelope of the performance of neutron production facilities currently available. The team has proposed a novel design that increases significantly the ability to evacuate high rates of heat deposition, whilst remaining within the bounds of existing well-proven materials and manufacturing technologies. Advances in computational power have enabled the project to adopt a lean approach based on CFD/FEM analysis instead of relying on intermediary testing of components, resulting in significant savings.

At the time of writing, the prototype has been operated successfully in several test runs at IPUL, gathering operational experience and a considerable volume of experimental data. Details of the test setup, the analysis and interpretation of the collected data will follow this first publication [12]. First indications are that the target performed as expected from the analysis, with low hydraulic losses and no significant vibrations.

\section{Acknowledgments}

The work was performed in the context of the EURISOL initiative. Special thanks go to Dr. F. Gröschel, former director of advanced projects at PSI and currently heading the IFMIF target group at the Rokkasho Fusion research centre in Japan, E. Manfrin of PSI for technical support, interns Q. Prétet and E. Brun of the
ICAM Engineering School in Toulouse, France and V. Geza of the University of Latvia. Yacine Kadi, Mats Lindroos and Yorick Blumenfeld representing project management at CERN in Geneva are also thanked for the computational resources generously allocated to the project.

We acknowledge the financial support of the European Commission under the Sixth Framework Program "Research Infrastructure Action-Structuring the European Research Area" EURISOL DS Project Contract no. 515768 RIDS. The EC is not liable for any use that may be made of the information contained herein.

\section{References}

[1] Y. Takeda, K. Samec, in: ASME Experimental and Numerical Flow visualization Proceedings, vol. 128, 1991, p. 47.

[2] W. Wagner, F. Groeschel, K. Thomsen, H. Heyck, J. Nucl. Mater 377 (2008) 12.

[3] Y. Blumenfeld, EURISOL: review of accelerators for radioactive beams, in: Proceedings of EPAC08, Genoa, Italy, June 2007.

[4] T.V. Dury, Nucl. Instr. and Meth. A 562 (2) (2006) 859.

[5] K. Thomsen, Nucl. Instr. and Meth. A 575 (3) (2007) 347.

[6] Q. Prétet, R. Milenkovic, B. Smith, Stress analysis of the EURISOL DS target, PSI Technical Note N TM-34-02, October.2006.

[7] M. Ashrafi-Nik, Thermo hydraulic optimisation of the EURISOL DS target, PSI Technical Note N TM-34-06-0, December 2006.

[8] K. Samec, Design of the EURISOL converter target, PSI Technical Note N TM-34-07-05, July 2007.

[9] R. Milenkovic, V. Geza, Hydraulic simulation of the flow condition at the inlet of the beam entrance window of the EURISOL converter target, PSI Technical Note N EURISOL-MR34-004/0, May 2008.

[10] K. Samec, M. Lindroos, Y. Kadi, EURISOL-DS multi-MW target fluid-structure interaction, CERN Technical Note N EURISOL-DS/TASK2/TN-02-25-20090034, January 2009.

[11] R. Milenkovic, S. Dementjev, K. Samec, E Platacis, A. Zik, A. Flerov, K. Thomsen, Structural-hydraulic test of the liquid metal EURISOL target mock-up, Nucl. Instr. and Meth. A, accepted for publication.

[12] R. Milenkovic, S. Dementjevs, K. Samec, B. Sigg, Wavelet analysis of experimental results for coupled structural-hydraulic behaviour of the EURISOL target mock-up, Nucl. Instr. and Meth. A, accepted for publication. 